\title{
Existence of the Mild Solutions for Delay Fractional Integrodifferential Equations with Almost Sectorial Operators
}

\author{
Fang Li \\ School of Mathematics, Yunnan Normal University, Kunming 650092, China \\ Correspondence should be addressed to Fang Li, fangli860@gmail.com
}

Received 1 February 2012; Accepted 8 June 2012

Academic Editor: Detlev Buchholz

Copyright (C) 2012 Fang Li. This is an open access article distributed under the Creative Commons Attribution License, which permits unrestricted use, distribution, and reproduction in any medium, provided the original work is properly cited.

This paper is concerned with the existence of mild solutions for the fractional integrodifferential equations with finite delay and almost sectorial operators in a separable Banach space $X$. We obtain existence theorem for mild solutions to the above-mentioned equations, by means of measure of noncompactness and the resolvent operators associated with almost sectorial operators. As an application, the existence of mild solutions for some integrodifferential equation is obtained.

\section{Introduction}

Fractional differential and integrodifferential equations have received increasing attention during recent years and have been studied extensively (see, e.g., [1-15] and references therein) since they are playing an increasingly important role in engineering, physics, electrolysis chemical, fractional biological neurons, condensate physics, statistical mechanics, and so on.

Moreover, the Cauchy problem for various delay equations in Banach spaces has been receiving more and more attention during the past decades (see, e.g., $[3,4,9,10,14,16-20]$ and references therein).

We mention that much of the previous research on the fractional equations was done provided that the operator in the linear part is the infinitesimal generator of a strongly continuous operator semigroup, a compact semigroup, or an analytic semigroup, or is a Hille-Yosida operator (see, e.g., [1-4, 8-10, 12-14]). However, as presented in [15, Examples 1.1 and 1.2], for which the resolvent operators do not satisfy the required estimate to be a sectorial operator. Von Wahl in [21] first introduced examples of almost sectorial 
operators which are not sectorial. Recently, the study of evolution equations involving almost sectorial operators has been investigated extensively. However, much less is known about the fractional evolution equations with almost sectorial operators (see [15] and the references therein). equations:

In this paper, we are concerned with the following fractional integrodifferential

$$
\begin{gathered}
{ }^{c} D^{q} u(t)=A u(t)+f\left(t, u_{t}\right)+\int_{0}^{t} g\left(t, s, u_{s}\right) d s, \quad t \in(0, T], \\
u(t)=\phi(t), \quad t \in[-r, 0],
\end{gathered}
$$

where $T>0,0<q<1$, and $0<r<\infty$. The fractional derivative is understood here in the Caputo sense. $X$ is a separable Banach space. $A$ is an almost sectorial operator to be introduced later. Here $f:[0, T] \times C([-r, 0], X) \rightarrow X, g: \Lambda \times C([-r, 0], X) \rightarrow X(\Lambda=$ $\{(t, s) \in[0, T] \times[0, T]: t \geq s\}), \phi \in C([-r, 0], X)$, where $C([a, b], X)$ denotes the space of all continuous functions from $[a, b]$ to $X$.

For any continuous function $v$ defined on $[-r, T]$ and any $t \in[0, T]$, we denote by $u_{t}$ the element of $C([-r, 0], X)$ defined by $u_{t}(\theta)=u(t+\theta), \theta \in[-r, 0]$.

Our paper is organized as follows. In Section 2, we give out some preliminaries about fractional order operator, measure of noncompactness, and almost sectorial operators. The existence result will be established in Section 3. In Section 4, an example is given to show the application of the abstract result.

\section{Preliminaries}

Throughout this paper, we denote by $X$ a separable Banach space with norm $\|\cdot\|$. For a linear operator $A$, we denote by $D(A)$ the domain of $A$, by $\rho(A)$ the resolvent set of $A$, and by $R(z ; A)=(z I-A)^{-1}, z \in \rho(A)$ the resolvent of $A$. Moreover, we denote by $L(X)$ the Banach space of all linear and bounded operators on $X$ and by $C([a, b], X)$ the space of all $X$-valued continuous functions on $[a, b]$ with the supremum norm as follows:

$$
\|x\|_{[a, b]}=\|x\|_{C([a, b], X)}=\sup \{\|x(t)\|: t \in[a, b]\}, \quad \text { for any } x \in C([a, b], X) .
$$

Moreover, we abbreviate $\|u\|_{L^{p}\left([0, T], \mathbf{R}^{+}\right)}$with $\|u\|_{L^{p}}$ for any $u \in L^{p}\left([0, T], \mathbf{R}^{+}\right)$.

Let us recall the following known definitions. For more details, see $[7,11]$.

Definition 2.1 (see [11]). The fractional integral of order $q$ with the lower limit zero for a function $f \in A C[0, \infty)$ is defined as

$$
I^{q} f(t)=\frac{1}{\Gamma(q)} \int_{0}^{t}(t-s)^{q-1} f(s) d s, \quad t>0,0<q<1
$$

provided that the right side is pointwise defined on $[0, \infty)$, where $\Gamma(\cdot)$ is the gamma function. 
Definition 2.2 (see [11]). The Riemann-Liouville derivative of order $q$ with the lower limit zero for a function $f \in A C[0, \infty)$ can be written as

$$
{ }^{L} D^{q} f(t)=\frac{1}{\Gamma(1-q)} \frac{d}{d t} \int_{0}^{t}(t-s)^{-q} f(s) d s, \quad t>0,0<q<1 .
$$

Definition 2.3 (see [11]). The Caputo derivative of order $q$ for a function $f \in A C[0, \infty)$ can be written as

$$
{ }^{c} D^{q} f(t)={ }^{L} D^{q}(f(t)-f(0)), \quad t>0,0<q<1 .
$$

Remark 2.4. (1) If $f(t) \in C^{1}[0, \infty)$, then

$$
{ }^{c} D^{q} f(t)=\frac{1}{\Gamma(1-q)} \int_{0}^{t}(t-s)^{-q} f^{\prime}(s) d s=I^{1-q} f^{\prime}(t), \quad t>0,0<q<1 ;
$$

(2) the Caputo derivative of a constant is equal to zero.

We will need the following facts from the theory of measures of noncompactness and condensing maps (see, e.g., $[22,23])$.

Definition 2.5. Let $E$ be a Banach space, $2^{E}$ the family of all nonempty subsets of $E,(\mathcal{A}, \geq)$ a partially ordered set and $v: 2^{E} \rightarrow \mathcal{A}$. If for every $\Omega \in 2^{E}$ :

$$
v(\overline{c O}(\Omega))=v(\Omega)
$$

then we say that $v$ is a measure of noncompactness (MNC) in $E$.

A MNC $v$ is called:

(i) monotone if $\Omega_{0}, \Omega_{1} \in 2^{E}, \Omega_{0} \subset \Omega_{1}$ implies $v\left(\Omega_{0}\right) \leq v\left(\Omega_{1}\right)$;

(ii) nonsingular if $v\left(\left\{a_{0}\right\} \cup \Omega\right)=v(\Omega)$ for every $a_{0} \in E, \Omega \in 2^{E}$;

(iii) invariant with respect to union with compact sets if $v(\{D\} \cup \Omega)=v(\Omega)$ for every relatively compact set $D \subset E, \Omega \in 2^{E}$.

If $\mathcal{A}$ is a cone in a normed space, we say that the MNC $v$ is;

(iv) algebraically semiadditive if $v\left(\Omega_{0}+\Omega_{1}\right) \leq v\left(\Omega_{0}\right)+v\left(\Omega_{1}\right)$ for each $\Omega_{0}, \Omega_{1} \in 2^{E}$;

(v) regular if $v(\Omega)=0$ is equivalent to the relative compactness of $\Omega$;

(vi) real if $\mathcal{A}$ is $[0,+\infty)$ with the natural order.

As an example of the MNC possessing all these properties, we may consider the Hausdorff MNC:

$$
\chi(\Omega)=\inf \{\varepsilon>0: \Omega \text { has a finite } \varepsilon \text {-net }\} .
$$

Now, let $G:[0, h] \rightarrow 2^{E}$ be a multifunction. It is called:

(i) integrable, if it admits a Bochner integrable selection $\mathfrak{g}:[0, h] \rightarrow E, \mathfrak{g}(t) \in G(t)$ for a.e. $t \in[0, h]$; 
(ii) integrably bounded, if there exists a function $\rho \in L^{1}([0, h], E)$ such that

$$
\|G(t)\|:=\sup \{\|\mathfrak{g}\|: \mathfrak{g} \in G(t)\} \leq \rho(t) \text { a.e. } t \in[0, h] .
$$

We present the following assertion about $x$-estimates for a multivalued integral [23, Theorem 4.2.3].

Proposition 2.6. For an integrable, integrably bounded multifunction $G:[0, h] \rightarrow 2^{X}$, where $X$ is a separable Banach space, let

$$
\chi(G(t)) \leq \mathfrak{q}(t), \quad \text { for a.e. } t \in[0, h]
$$

where $\mathfrak{q} \in L_{+}^{1}([0, h])$. Then, $X\left(\int_{0}^{t} G(s) d s\right) \leq \int_{0}^{t} \mathfrak{q}(s) d s$ for all $t \in[0, h]$.

Let $E$ be a Banach space, $v$ a monotone nonsingular MNC in $E$.

Definition 2.7. A continuous map $\mathfrak{F}: Y \subseteq E \rightarrow E$ is called condensing with respect to a MNC $\mathcal{v}$ (or $\mathcal{v}$-condensing) if for every bounded set $\Omega \subseteq Y$ which is not relatively compact, we have

$$
v(\mathfrak{F}(\Omega)) \nsupseteq v(\Omega) .
$$

The following fixed point principle (see, e.g., $[22,23]$ ) will be used later.

Theorem 2.8. Let $\mathfrak{M}$ be a bounded convex closed subset of $E$ and $\mathfrak{F}: \mathfrak{M} \rightarrow \mathfrak{M}$ a $\mathcal{v}$-condensing map. Then, Fix $\mathfrak{F}=\{x: x=\mathfrak{F}(x)\}$ is nonempty.

Theorem 2.9. Let $V \subset E$ be a bounded open neighborhood of zero and $\mathfrak{F}: \bar{V} \rightarrow E$ a v-condensing map satisfying the boundary condition:

$$
x \neq \bar{\lambda} \mathfrak{F}(x),
$$

for all $x \in \partial V$ and $0<\bar{\lambda} \leq 1$. Then, Fix $\mathfrak{F}$ is a nonempty compact set.

To prove the main result, we will need the following generalization of Gronwall's lemma for singular kernels [24, Lemma 7.1.1].

Lemma 2.10. Let $x, y:[0, T] \rightarrow[0,+\infty)$ be continuous functions. If $y(\cdot)$ is nondecreasing and there are constants $\mathfrak{a}>0$ and $0<\vartheta<1$ such that

$$
x(t) \leq y(t)+\mathfrak{a} \int_{0}^{t}(t-s)^{-\vartheta} x(s) d s,
$$

then there exists a constant $\mathcal{\kappa}=\kappa(\vartheta)$ such that

$$
x(t) \leq y(t)+\kappa \mathfrak{a} \int_{0}^{t}(t-s)^{-\vartheta} y(s) d s, \quad \text { for each } t \in[0, T]
$$


Next, we recall the knowledge of almost sectorial operator, for more details, we refer to $[25,26]$.

Let $-1<\gamma<0$ and $S_{\mu}^{0}$ with $0<\mu<\pi$ be the open sector:

$$
\{z \in \mathbf{C} \backslash\{0\}:|\arg z|<\mu\},
$$

and $S_{\mu}$ its closure, that is,

$$
S_{\mu}=\{z \in \mathbf{C} \backslash\{0\}:|\arg z| \leq \mu\} \cup\{0\} .
$$

Let us recall the following definition.

Definition 2.11. Let $-1<\gamma<0$ and $0<\omega<\pi / 2$. By $\Theta_{\omega}^{\gamma}(X)$, we denote the family of all linear closed operators $A: D(A) \subset X \rightarrow X$ which satisfy:

(1) $\sigma(A) \subset S_{\omega}=\{z \in \mathbf{C} \backslash\{0\}:|\arg z| \leq \omega\} \cup\{0\}$;

(2) for every $\omega<\mu<\pi$, there exists a constant $C_{\mu}$ such that

$$
\|R(z ; A)\|_{L(X)} \leq C_{\mu}|z|^{\gamma}, \quad \text { for all } z \in \mathbf{C} \backslash S_{\mu} \text {. }
$$

A linear operator $A$ will be called an almost sectorial operator on $X$ if $A \in \Theta_{\omega}^{\gamma}(X)$.

Remark 2.12. Let $A \in \Theta_{\omega}^{\gamma}(X)$, then the definition implies that $0 \in \rho(A)$.

Remark 2.13 (see [15]). From [26], note in particular that if $A \in \Theta_{\omega}^{\gamma}(X)$, then $A$ generates a semigroup $T(t)$ with a singular behavior at $t=0$ in a sense, called semigroup of growth $1+\gamma$. Moreover, the semigroup $T(t)$ is analytic in an open sector of the complex plane $\mathbf{C}$, but the strong continuity fails at $t=0$ for data which are not sufficiently smooth.

We denote the semigroup associated with $A$ by $T(t)$. For $t \in S_{\pi / 2-\omega^{\prime}}^{0}$

$$
T(t)=e^{-t z}(A)=\frac{1}{2 \pi i} \int_{\Gamma_{\theta}} e^{-t z} R(z ; A) d z
$$

forms an analytic semigroup of growth order $1+\gamma$, where $\omega<\theta<\mu<\pi / 2-|\arg t|$, the integral contour $\Gamma_{\theta}:=\left\{\mathbf{R}^{+} e^{i \theta}\right\} \cup\left\{\mathbf{R}^{+} e^{-i \theta}\right\}$ is oriented counter-clockwise $([15,26])$.

We have the following proposition on $T(t)$ [26, Theorem 3.9].

Proposition 2.14. Let $A \in \Theta_{\omega}^{\gamma}(X)$ with $-1<\gamma<0$ and $0<\omega<\pi / 2$. Then the following properties remain true:

(i) $T(t)$ is analytic in $S_{\pi / 2-\omega}^{0}$ and

$$
\frac{d^{n}}{d t^{n}} T(t)=(-A)^{n} T(t), \quad \text { for all } t \in S_{\pi / 2-\omega^{0}}^{0}
$$


(ii) the functional equation $T(s+t)=T(s) T(t)$ for all $s, t \in S_{\pi / 2-\omega}^{0}$ holds;

(iii) there exists a constant $C_{0}=C_{0}(\gamma)>0$ such that

$$
\|T(t)\|_{L(X)} \leq C_{0} t^{-\gamma-1}, \text { for all } t>0 ;
$$

(iv) if $\beta>1+\gamma$, then $D\left(A^{\beta}\right) \subset \Sigma_{T}=\left\{x \in X: \lim _{t \rightarrow 0 ; t>0} T(t) x=x\right\}$. or $s=0$.

Clearly, we note that the condition (ii) of the Proposition 2.14 does not satisfy for $t=0$

The relation between the resolvent operators of $A$ and the semigroup $T(t)$ is characterized by.

Proposition 2.15 (see [26, Theorem 3.13]). Let $A \in \Theta_{\omega}^{\gamma}(X)$ with $-1<\gamma<0$ and $0<\omega<\pi / 2$. Then for every $\lambda \in \mathrm{C}$ with $\operatorname{Re} \lambda>0$, one has

$$
R(\lambda ; A)=\int_{0}^{\infty} e^{-\lambda t} T(t) d t
$$

Based on the work in [15], we define operator families $\left.\left\{\mathcal{S}_{q}(t)\right\}\right|_{t \in S_{\pi / 2-\omega}^{0}}$ and $\left.\left\{p_{q}(t)\right\}\right|_{t \in S_{\pi / 2-\omega}^{0}}$ by

$$
\begin{gathered}
\mathcal{S}_{q}(t) x=\int_{0}^{\infty} \Psi_{q}(\sigma) T\left(\sigma t^{q}\right) x d \sigma, \quad t \in S_{\pi / 2-\omega^{\prime}}^{0} x \in X, \\
P_{q}(t) x=\int_{0}^{\infty} q \sigma \Psi_{q}(\sigma) T\left(\sigma t^{q}\right) x d \sigma, \quad t \in S_{\pi / 2-\omega^{\prime}}^{0} x \in X,
\end{gathered}
$$

where $\Psi_{q}(z)$ with $0<q<1$ is a function of Wright-type (cf. e.g., [15]) as follows:

$$
\Psi_{q}(z):=\sum_{n=0}^{\infty} \frac{(-z)^{n}}{n ! \Gamma(-q n+1-q)}=\frac{1}{\pi} \sum_{n=1}^{\infty} \frac{(-z)^{n}}{(n-1) !} \Gamma(n q) \sin (n \pi q), \quad z \in \mathbf{C} .
$$

We collect some basic properties on $\Psi_{q}(z)$. For more details, we refer to $([7,11,15,25])$.

Proposition 2.16. For $-1<\tilde{r}<\infty, \lambda>0$, the following results hold:

(1) $\Psi_{q}(t) \geq 0, t>0$;

(2) $\int_{0}^{\infty} q / t^{q+1} \Psi_{q}\left(1 / t^{q}\right) e^{-\lambda t} d t=e^{-\lambda^{q}}$;

(3) $\int_{0}^{\infty} \Psi_{q}(t) t^{\tilde{r}} d t=\Gamma(1+\tilde{r}) / \Gamma(1+q \tilde{r})$.

Theorem 2.17 (see [15, Theorem 3.1]). For each fixed $t \in S_{\pi / 2-\omega^{\prime}}^{0} S_{q}(t)$ and $p_{q}(t)$ are linear and bounded operators on X. Moreover, for all $t>0,-1<\gamma<0,0<q<1$,

$$
\begin{gathered}
\left\|\mathcal{S}_{q}(t) x\right\| \leq \frac{C_{0} \Gamma(-\gamma)}{\Gamma(1-q(1+\gamma))} t^{-q(1+\gamma)}\|x\|, \quad x \in X, \\
\left\|D_{q}(t) x\right\| \leq \frac{q C_{0} \Gamma(1-\gamma)}{\Gamma(1-q \gamma)} t^{-q(1+\gamma)}\|x\|, \quad x \in X .
\end{gathered}
$$


Theorem 2.18 (see [15, Theorem 3.2]). For $t>0, \mathcal{S}_{q}(t)$ and $p_{q}(t)$ are continuous in the uniform operator topology. Moreover, for every $\tilde{r}>0$, the continuity is uniform on $[\tilde{r}, \infty)$.

Remark 2.19 (see [15, Theorem 3.4]). Let $\beta>1+\gamma$. Then for all $x \in D\left(A^{\beta}\right)$,

$$
\lim _{t \rightarrow 0 ; t>0} \mathcal{S}_{q}(t) x=x
$$

Next, we will present the definition of mild solution of problem (1.1).

According to Definitions 2.1-2.3, we can rewrite problem (1.1) in the equivalent integral equation:

$$
\begin{gathered}
u(t)=\phi(0)+\frac{1}{\Gamma(q)} \int_{0}^{t}(t-s)^{q-1}\left[A u(s)+f\left(s, u_{s}\right)+a(u)(s)\right] d s, \quad t \in(0, T] \\
u(t)=\phi(t), \quad t \in[-r, 0]
\end{gathered}
$$

provided that the integral in (2.25) exists, where

$$
a(u)(t)=\int_{0}^{t} g\left(t, s, u_{s}\right) d s
$$

Set

$$
\widehat{u}(\lambda)=\int_{0}^{\infty} e^{-\lambda t} u(t) d t, \quad \widehat{f}(\lambda)=\int_{0}^{\infty} e^{-\lambda t} f\left(t, u_{t}\right) d t, \quad \widehat{g}(\lambda)=\int_{0}^{\infty} e^{-\lambda t} a(u)(t) d t,
$$

formally applying the Laplace transform to (2.25), we get

$$
\widehat{u}(\lambda)=\frac{1}{\lambda} \phi(0)+\frac{1}{\lambda^{q}} A \widehat{u}(\lambda)+\frac{1}{\lambda^{q}}[\widehat{f}(\lambda)+\widehat{g}(\lambda)],
$$

then

thus

$$
\left(\lambda^{q}-A\right) \widehat{u}(\lambda)=\lambda^{q-1} \phi(0)+[\widehat{f}(\lambda)+\widehat{g}(\lambda)]
$$

$$
\begin{aligned}
\widehat{u}(\lambda) & =\lambda^{q-1}\left(\lambda^{q}-A\right)^{-1} \phi(0)+\left(\lambda^{q}-A\right)^{-1}[\widehat{f}(\lambda)+\widehat{g}(\lambda)] \\
& =\lambda^{q-1} \int_{0}^{\infty} e^{-\lambda^{q} s} T(s) \phi(0) d s+\int_{0}^{\infty} e^{-\lambda^{q} s} T(s)[\widehat{f}(\lambda)+\widehat{g}(\lambda)] d s
\end{aligned}
$$

provided that the integral in (2.30) exists.

Then, using Proposition 2.16 (2), we have

$$
\begin{aligned}
\lambda^{q-1} \int_{0}^{\infty} e^{-\lambda^{q}} T(s) \phi(0) d s & =q \int_{0}^{\infty}(\lambda t)^{q-1} e^{-(\lambda t)^{q}} T\left(t^{q}\right) \phi(0) d t \\
& =-\frac{1}{\lambda} \int_{0}^{\infty}\left(\frac{d}{d t} e^{-(\lambda t)^{q}}\right) T\left(t^{q}\right) \phi(0) d t
\end{aligned}
$$




$$
\begin{aligned}
& =\int_{0}^{\infty} \int_{0}^{\infty} \frac{q}{\sigma^{q}} \Psi_{q}\left(\frac{1}{\sigma^{q}}\right) e^{-\lambda t \sigma} T\left(t^{q}\right) \phi(0) d \sigma d t \\
& =\int_{0}^{\infty} e^{-\lambda t}\left[\int_{0}^{\infty} \frac{q}{\sigma^{q+1}} \Psi_{q}\left(\frac{1}{\sigma^{q}}\right) T\left(\frac{t^{q}}{\sigma^{q}}\right) \phi(0) d \sigma\right] d t \\
& =\int_{0}^{\infty} e^{-\lambda t}\left[\int_{0}^{\infty} \Psi_{q}(\tau) T\left(t^{q} \tau\right) \phi(0) d \tau\right] d t \\
\int_{0}^{\infty} e^{-\lambda{ }^{q} s} T(s) \widehat{f}(\lambda) d s & =\int_{0}^{\infty} e^{-(\lambda \tau)^{q}} q \tau^{q-1} T\left(\tau^{q}\right)\left(\int_{0}^{\infty} e^{-\lambda t} f\left(t, u_{t}\right) d t\right) d \tau \\
& =\int_{0}^{\infty} \int_{0}^{\infty} e^{-\lambda \tau \sigma} \frac{q}{\sigma^{q+1}} \Psi_{q}\left(\frac{1}{\sigma^{q}}\right) q \tau^{q-1} T\left(\tau^{q}\right)\left(\int_{0}^{\infty} e^{-\lambda t} f\left(t, u_{t}\right) d t\right) d \sigma d \tau \\
& =q^{2} \int_{0}^{\infty} \int_{0}^{\infty} e^{-\lambda \theta} \frac{\theta^{q-1}}{\sigma^{2 q+1}} \Psi_{q}\left(\frac{1}{\sigma^{q}}\right) T\left(\frac{\theta^{q}}{\sigma^{q}}\right)\left(\int_{0}^{\infty} e^{-\lambda t} f\left(t, u_{t}\right) d t\right) d \theta d \sigma \\
& =q^{2} \int_{0}^{\infty}\left(\int_{0}^{\infty} \int_{t}^{\infty} e^{-\lambda \tau} \frac{(\tau-t)^{q-1}}{\sigma^{2 q+1}} \Psi_{q}\left(\frac{1}{\sigma^{q}}\right) T\left(\frac{(\tau-t)^{q}}{\sigma^{q}}\right) f\left(t, u_{t}\right) d \tau d t\right) d \sigma \\
& =q^{2} \int_{0}^{\infty}\left(\int_{0}^{\infty} \int_{0}^{\tau} e^{-\lambda \tau} \frac{(\tau-t)^{q-1}}{\sigma^{2 q+1}} \Psi_{q}\left(\frac{1}{\sigma^{q}}\right) T\left(\frac{(\tau-t)^{q}}{\sigma^{q}}\right) f\left(t, u_{t}\right) d t d \tau\right) d \sigma \\
& =\int_{0}^{\infty} e^{-\lambda t}\left[q \int_{0}^{t} \int_{0}^{\infty}(t-s)^{q-1} \sigma \Psi_{q}(\sigma) T\left((t-s)^{q} \sigma\right) f\left(s, u_{s}\right) d \sigma d s\right] d t .
\end{aligned}
$$

Similarly, we have

$$
\int_{0}^{\infty} e^{-\lambda^{q} s} T(s) \widehat{g}(\lambda) d s=\int_{0}^{\infty} e^{-\lambda t}\left[q \int_{0}^{t} \int_{0}^{\infty}(t-s)^{q-1} \sigma \Psi_{q}(\sigma) T\left((t-s)^{q} \sigma\right) a(u)(s) d \sigma d s\right] d t
$$

Thus, from (2.30)-(2.32), we obtain

$$
\begin{aligned}
\widehat{u}(\lambda)=\int_{0}^{\infty} e^{-\lambda t}\left[\int_{0}^{\infty} \Psi_{q}(\tau) T\left(t^{q} \tau\right) \phi(0) d \tau\right. \\
\quad+q \int_{0}^{t} \int_{0}^{\infty}(t-s)^{q-1} \sigma \Psi_{q}(\sigma) T\left((t-s)^{q} \sigma\right) f\left(s, u_{s}\right) d \sigma d s \\
\left.\quad+q \int_{0}^{t} \int_{0}^{\infty}(t-s)^{q-1} \sigma \Psi_{q}(\sigma) T\left((t-s)^{q} \sigma\right) a(u)(s) d \sigma d s\right] d t .
\end{aligned}
$$


We invert the last Laplace transform to obtain

$$
\begin{aligned}
u(t)= & \int_{0}^{\infty} \Psi_{q}(\tau) T\left(t^{q} \tau\right) \phi(0) d \tau \\
& +q \int_{0}^{t} \int_{0}^{\infty}(t-s)^{q-1} \sigma \Psi_{q}(\sigma) T\left((t-s)^{q} \sigma\right) f\left(s, u_{s}\right) d \sigma d s \\
& +q \int_{0}^{t} \int_{0}^{\infty}(t-s)^{q-1} \sigma \Psi_{q}(\sigma) T\left((t-s)^{q} \sigma\right) a(u)(s) d \sigma d s \\
= & \mathcal{S}_{q}(t) \phi(0)+\int_{0}^{t}(t-s)^{q-1} p_{q}(t-s) f\left(s, u_{s}\right) d s \\
& +\int_{0}^{t}(t-s)^{q-1} p_{q}(t-s) a(u)(s) d s .
\end{aligned}
$$

Then from the above induction, when $\phi(0) \in D\left(A^{\beta}\right)$ with $\beta>1+\gamma$, we can give the following definition of the mild solution of (1.1).

Definition 2.20. A continuous function $u:[-r, T] \rightarrow X$ satisfying the equation:

$$
u(t)= \begin{cases}\phi(t), & t \in[-r, 0], \\ \mathcal{S}_{q}(t) \phi(0)+\int_{0}^{t}(t-s)^{q-1} p_{q}(t-s) f\left(s, u_{s}\right) d s & \\ \quad+\int_{0}^{t}(t-s)^{q-1} p_{q}(t-s) a(u)(s) d s, & t \in[0, T]\end{cases}
$$

is called a mild solution of (1.1).

Remark 2.21 (see [15], Remark 4.1). (1) In general, since the operator $\mathcal{S}_{q}(t)$ is singular at $t=0$, solutions to problem (1.1) are assumed to have the same kind of singularity at $t=0$ as the operator $\mathcal{S}_{q}(t)$.

(2) When $\phi(0) \in D\left(A^{\beta}\right)$ with $\beta>1+\gamma$, it follows from Remark 2.19 that the mild solution is continuous at $t=0$.

\section{Main Result}

Throughout this section, let $A$ be an operator in the class $\Theta_{\omega}^{\gamma}(X)$ and $-1<\gamma<0,0<\omega<$ $\pi / 2$. Moreover, we require the following assumptions:

(Hf) (1) $f:[0, T] \times C([-r, 0], X) \rightarrow X$ satisfies $f(\cdot, y):[0, T] \rightarrow X$ is measurable for all $y \in C([-r, 0], X)$ and $f(t, \cdot): C([-r, 0], X) \rightarrow X$ is continuous for a.e. $t \in[0, T]$, and there exists a positive function $\mu(\cdot) \in L^{p}\left([0, T], \mathbf{R}^{+}\right)(p>-1 /(q \gamma)>1 / q>1)$ such that

$$
\|f(t, y)\| \leq \mu(t)\|y\|_{[-r, 0]}
$$

for almost all $t \in[0, T]$; 
(2) there exists a nondecreasing function $\eta \in L^{p}\left([0, T], \mathbf{R}^{+}\right)$such that for any bounded set $D \subset C([-r, 0], X)$ :

$$
\chi(f(t, D)) \leq \eta(t) \sup _{\theta \in[-r, 0]} x(D(\theta)), \quad \text { a.e. } t \in[0, T] .
$$

(Hg) (1) $g: \Lambda \times C([-r, 0], X) \rightarrow X$ and $g(t, s, \cdot): C([-r, 0], X) \rightarrow X$ is continuous for a.e. $(t, s) \in \Lambda$, and for each $y \in C([-r, 0], X)$, the function $g(\cdot, \cdot, y)$ : $\Lambda \rightarrow X$ is measurable. Moreover, there exists a function $m: \Lambda \rightarrow \mathbf{R}^{+}$with $\sup _{t \in[0, T]} \int_{0}^{t} m(t, s) d s:=m^{*}<\infty$ such that

$$
\|g(t, s, y)\| \leq m(t, s)\|y\|_{[-r, 0]},
$$

for almost all $(t, s) \in \Lambda$;

(2) for any bounded set $D \subset C([-r, 0], X)$, there exists a function $\xi: \Lambda \rightarrow \mathbf{R}^{+}$such that

$$
\chi(g(t, s, D)) \leq \xi(t, s) \sup _{\theta \in[-r, 0]} x(D(\theta))
$$

where $\sup _{t \in[0, T]} \int_{0}^{t} \xi(t, s) d s:=\xi^{*}<\infty$.

Theorem 3.1. Let $A \in \Theta_{\omega}^{\gamma}(\mathrm{X})$ with $-1<\gamma<0,0<\omega<\pi / 2$. Assume that (Hf) and (Hg) are satisfied. Then for every $\phi(0) \in D\left(A^{\beta}\right)$ with $\beta>1+\gamma$, the mild solution set of problem (1.1) is a nonempty compact subset of the space $C([-r, T], X)$, provided that

$$
M\left(l_{p, q} T^{-(1+p q r) / p}\|\mu\|_{L^{p}}+\frac{-m^{*} T^{-q \gamma}}{q \gamma}\right)<1
$$

where $M:=\left(q C_{0} \Gamma(1-\gamma)\right) / \Gamma(1-q \gamma)$ and $l_{p, q}:=((p-1) /(-p q \gamma-1))^{(p-1) / p}$.

Proof. We define the operator $\mathcal{F}: C([-r, T], X) \rightarrow C([-r, T], X)$ in the following way:

$$
(\mathcal{F} u)(t)= \begin{cases}\phi(t), & t \in[-r, 0] \\ \mathcal{S}_{q}(t) \phi(0)+\int_{0}^{t}(t-s)^{q-1} p_{q}(t-s) f\left(s, u_{s}\right) d s & \\ \quad+\int_{0}^{t}(t-s)^{q-1} p_{q}(t-s) a(u)(s) d s, & t \in[0, T] .\end{cases}
$$

It is clear that the operator $\mp$ is well defined.

We define

$$
\widehat{\phi}(t)= \begin{cases}\phi(t), & t \in[-r, 0] \\ \mathcal{S}_{q}(t) \phi(0), & t \in[0, T] .\end{cases}
$$


Let $u(t)=v(t)+\widehat{\phi}(t)$. It is easy to see that $v$ satisfies $v_{0}=0$ and

$$
v(t)=\int_{0}^{t}(t-s)^{q-1} p_{q}(t-s) f\left(s, v_{s}+\widehat{\phi}_{s}\right) d s+\int_{0}^{t}(t-s)^{q-1} D_{q}(t-s) a(v+\widehat{\phi})(s) d s, \quad t \in[0, T]
$$

if and only if $u$ satisfies

$$
u(t)=\mathcal{S}_{q}(t) \phi(0)+\int_{0}^{t}(t-s)^{q-1} p_{q}(t-s) f\left(s, u_{s}\right) d s+\int_{0}^{t}(t-s)^{q-1} p_{q}(t-s) a(u)(s) d s, \quad t \in[0, T]
$$

and $u(t)=\phi(t), t \in[-r, 0]$.

Let $\widehat{\mathcal{F}}: C([-r, T], X) \rightarrow C([-r, T], X)$ be an operator defined by $(\hat{\mathcal{F}} v)(t)=0$ for $t \in$ $[-r, 0]$ and

$$
\begin{aligned}
(\widehat{\mathcal{F}} v)(t)= & \int_{0}^{t}(t-s)^{q-1} p_{q}(t-s) f\left(s, v_{s}+\widehat{\phi}_{s}\right) d s \\
& +\int_{0}^{t}(t-s)^{q-1} p_{q}(t-s) a(v+\widehat{\phi})(s) d s, \quad t \in[0, T] .
\end{aligned}
$$

Clearly the operator $\hat{\mathcal{F}}$ has a fixed point is equivalent to $\mathcal{F}$ having one.

We define $\mathcal{C}:=\left\{v \in C([-r, T], X): v_{0}=0\right\} \subset C([-r, T], X)$. Next we will prove that $\hat{\mathscr{f}}$ has a fixed point on $\mathcal{C}$.

Let $\left\{v^{n}\right\}_{n \in \mathbf{N}}$ be a sequence such that $v^{n} \rightarrow v$ in $\mathcal{C}$ as $n \rightarrow \infty$. Since $f$ satisfies (Hf)(1) and $g$ satisfies $(\mathrm{Hg})(1)$, for almost every $t \in[0, T]$ and $(t, s) \in \Lambda$, we get

$$
\begin{aligned}
f\left(t, v_{t}^{n}+\widehat{\phi}_{t}\right) & \longrightarrow f\left(t, v_{t}+\widehat{\phi}_{t}\right), \quad \text { as } n \longrightarrow \infty, \\
g\left(t, s, v_{s}^{n}+\widehat{\phi}_{s}\right) & \longrightarrow g\left(t, s, v_{s}+\widehat{\phi}_{s}\right), \quad \text { as } n \longrightarrow \infty .
\end{aligned}
$$

Noting that

$$
\begin{aligned}
\left\|v_{t}+\widehat{\phi}_{t}\right\|_{[-r, 0]} & \leq \sup _{t \in[-r, 0]}\|v(t)\|+\sup _{t \in[0, T]}\|v(t)\|+\sup _{t \in[-r, 0]}\|\widehat{\phi}(t)\|+\sup _{t \in[0, T]}\|\widehat{\phi}(t)\| \\
& =\sup _{t \in[0, T]}\|v(t)\|+\sup _{t \in[-r, 0]}\|\phi(t)\|+\sup _{t \in[0, T]}\left\|\mathcal{S}_{q}(t) \phi(0)\right\|:=\alpha .
\end{aligned}
$$

Therefore,

$$
\begin{gathered}
\left\|f\left(t, v_{t}+\widehat{\phi}_{t}\right)\right\| \leq \mu(t)\left\|v_{t}+\widehat{\phi}_{t}\right\|_{[-r, 0]} \leq \alpha \mu(t), \\
\|a(v+\widehat{\phi})(t)\| \leq \int_{0}^{t}\left\|g\left(t, s, v_{s}+\widehat{\phi}_{s}\right)\right\| d s \leq \int_{0}^{t} m(t, s)\left\|v_{s}+\widehat{\phi}_{s}\right\|_{[-r, 0]} d s \leq m^{*} \alpha .
\end{gathered}
$$


Since $v^{n} \rightarrow v$ in $\mathcal{C}$, it follows that there exists $\varepsilon>0$ such that $\left\|v^{n}-v\right\|_{[0, T]} \leq \varepsilon$ for $n$ sufficiently large. Moreover, noting that $\left\|v_{t}^{n}-v_{t}\right\|_{[-r, 0]} \leq\left\|v^{n}-v\right\|_{[0, T]}$, we have

$$
\begin{aligned}
\left\|f\left(t, v_{t}^{n}+\widehat{\phi}_{t}\right)-f\left(t, v_{t}+\widehat{\phi}_{t}\right)\right\| & \leq \mu(t)\left\|v_{t}^{n}+\widehat{\phi}_{t}\right\|_{[-r, 0]}+\alpha \mu(t) \\
& \leq \mu(t)\left\|v_{t}^{n}-v_{t}\right\|_{[-r, 0]}+2 \alpha \mu(t) \leq(\varepsilon+2 \alpha) \mu(t) .
\end{aligned}
$$

Similarly,

$$
\begin{gathered}
\left\|g\left(t, s, v_{s}^{n}+\widehat{\phi}_{s}\right)-g\left(t, s, v_{s}+\widehat{\phi}_{s}\right)\right\| \leq(\varepsilon+2 \alpha) m(t, s), \\
\left\|\int_{0}^{t} g\left(t, s, v_{s}^{n}+\widehat{\phi}_{s}\right) d s-\int_{0}^{t} g\left(t, s, v_{s}+\widehat{\phi}_{s}\right) d s\right\| \leq m^{*}(\varepsilon+2 \alpha) .
\end{gathered}
$$

It follows from the Lebesgue's Dominated Convergence Theorem that

$$
\left\|\int_{0}^{t} g\left(t, s, v_{s}^{n}+\widehat{\phi}_{s}\right) d s-\int_{0}^{t} g\left(t, s, v_{s}+\widehat{\phi}_{s}\right) d s\right\| \longrightarrow 0, \quad \text { as } n \longrightarrow \infty
$$

and from (2.23), we have

$$
\begin{gathered}
\left\|\int_{0}^{t}(t-s)^{q-1} p_{q}(t-s)\left[f\left(s, v_{s}^{n}+\widehat{\phi}_{s}\right)-f\left(s, v_{s}+\widehat{\phi}_{s}\right)\right] d s\right\| \\
+\left\|\int_{0}^{t}(t-s)^{q-1} p_{q}(t-s)\left[\int_{0}^{s} g\left(s, \tau, v_{\tau}^{n}+\widehat{\phi}_{\tau}\right) d \tau-\int_{0}^{s} g\left(s, \tau, v_{\tau}+\widehat{\phi}_{\tau}\right) d \tau\right] d s\right\| \| \\
\leq M \int_{0}^{t}(t-s)^{-q \gamma-1}\left[\left\|f\left(s, v_{s}^{n}+\widehat{\phi}_{s}\right)-f\left(s, v_{s}+\widehat{\phi}_{s}\right)\right\|\right. \\
\left.+\left\|\int_{0}^{s} g\left(s, \tau, v_{\tau}^{n}+\widehat{\phi}_{\tau}\right) d \tau-\int_{0}^{s} g\left(s, \tau, v_{\tau}+\widehat{\phi}_{\tau}\right) d \tau\right\|\right] d s \rightarrow 0,
\end{gathered}
$$

Therefore, we obtain that

$$
\lim _{n \rightarrow \infty}\left\|\hat{\mp} v^{n}-\hat{\mp} v\right\|_{[0, T]}=0
$$

then we see that $\hat{f}$ is continuous. 
Let us consider the MNC $v$ in the space $\mathcal{C}$ with values in the cone $\mathbf{R}_{+}^{3}$ of the following way: for every bounded subset $\Omega \subset \mathcal{C}$,

$$
v(\Omega)=\left(\sup _{t \in[-r, 0]} \chi(\Omega(t)), \Phi(\Omega), \bmod _{c}(\Omega)\right)
$$

where $\bmod _{c}(\Omega)$ is the module of equicontinuity of $\Omega$ given by

$$
\begin{gathered}
\bmod _{c}(\Omega)=\limsup _{\delta \rightarrow 0} \max _{v \in \Omega}\left\|v\left(t_{1}\right)-v\left(t_{2}\right)\right\|, \\
\Phi(\Omega)=\sup _{t \in[0, T]}\left(e^{-L t} \sup _{s \in[0, t]} X(\Omega(s))\right),
\end{gathered}
$$

where $L>0$ is a constant chosen so that

$$
\begin{gathered}
M \sup _{t \in[0, T]} \int_{0}^{t}(t-s)^{-q \gamma-1} \eta(s) e^{-L(t-s)} d s=L_{1}<1, \\
M \xi^{*} \sup _{t \in[0, T]} \int_{0}^{t}(t-s)^{-q \gamma-1} e^{-L(t-s)} d s=L_{2}<1 .
\end{gathered}
$$

Noting that for any $\psi \in L^{1}([0, T], X)$, we have

$$
\lim _{L \rightarrow+\infty} \sup _{t \in[0, T]} \int_{0}^{t} e^{-L(t-s)} \psi(s) d s=0,
$$

so, we can take the appropriate $L$ to satisfy (3.22) and (3.23).

Next, we show that the operator $\hat{\mathcal{F}}$ is $\mathcal{v}$-condensing on every bounded subset of $\mathcal{C}$.

Let $\Omega \subset \mathcal{C}$ be a nonempty, bounded set for which

$$
v(\hat{\mathcal{F}}(\Omega)) \geq v(\Omega) .
$$

Noting that

$$
\sup _{t \in[-r, 0]} x(\widehat{\mathscr{F}}(\Omega)(t))=0
$$

and (3.25), we can see that $\sup _{t \in[-r, 0]} \chi(\Omega(t))=0$.

Next, we estimate $\Phi(\Omega)$. For any $t \in[0, T]$, we set

$$
\widehat{\mathscr{f}}_{1}(\Omega)(t)=\left\{\int_{0}^{t}(t-s)^{q-1} p_{q}(t-s) f\left(s, v_{s}+\widehat{\phi}_{s}\right) d s: v \in \Omega\right\} .
$$


We consider the multifunction $s \in[0, t] \multimap H(s)$ :

$$
H(s)=\left\{(t-s)^{q-1} p_{q}(t-s) f\left(s, v_{s}+\widehat{\phi}_{s}\right): v \in \Omega\right\} .
$$

Obviously, $H$ is integrable, and from (2.23), (Hf) (1), and (3.13), it follows that $H$ is integrably bounded. Moreover, noting that (Hf)(2), we have the following estimate for a.e. $s \in[0, t]$ :

$$
\begin{aligned}
x(H(s)) & =x\left(\left\{(t-s)^{q-1} p_{q}(t-s) f\left(s, v_{s}+\widehat{\phi}_{s}\right): v \in \Omega\right\}\right) \\
& =x\left((t-s)^{q-1} p_{q}(t-s) f\left(s, \Omega_{s}+\widehat{\phi}_{s}\right)\right) \\
& \leq M(t-s)^{-q \gamma-1} \eta(s) \sup _{\tau \in[0, s]} x(\Omega(\tau)) \\
& =M(t-s)^{-q \gamma-1} \eta(s) e^{L s} e^{-L s} \sup _{\tau \in[0, s]} x(\Omega(\tau)) \\
& \leq M(t-s)^{-q \gamma-1} \eta(s) e^{L s} \Phi(\Omega) .
\end{aligned}
$$

Applying Proposition 2.6, we have

$$
\chi\left(\widehat{\Psi}_{1}(\Omega)(t)\right)=\chi\left(\int_{0}^{t} H(s) d s\right) \leq M \int_{0}^{t}(t-s)^{-q \gamma-1} \eta(s) e^{L s} d s \cdot \Phi(\Omega) .
$$

Therefore, from (3.22), we have

$$
\begin{aligned}
\sup _{t \in[0, T]}\left(e^{-L t} \sup _{s \in[0, t]} x\left(\hat{\mathscr{F}}_{1}(\Omega)(s)\right)\right) & \leq M \sup _{t \in[0, T]} \int_{0}^{t}(t-s)^{-q \gamma-1} \eta(s) e^{-L(t-s)} d s \cdot \Phi(\Omega) \\
& =L_{1} \Phi(\Omega) .
\end{aligned}
$$

Similarly, if we set

$$
\widehat{\mathscr{F}}_{2}(\Omega)(t)=\left\{\int_{0}^{t}(t-s)^{q-1} p_{q}(t-s) a(v+\widehat{\phi})(s) d s: v \in \Omega\right\}
$$

then we can see that the multifunction $s \in[0, t] \multimap \widetilde{H}(s)$,

$$
\widetilde{H}(s)=\left\{(t-s)^{q-1} p_{q}(t-s) a(v+\widehat{\phi})(s): v \in \Omega\right\}
$$


is integrable, and from (2.23), $(\mathrm{Hg})(1)$, and (3.14), it follows that $\widetilde{H}$ is integrably bounded. Moreover, noting that $(\mathrm{Hg})(2)$, Proposition 2.6, and (3.23), we have the following estimate for a.e. $s \in[0, t]$ :

$$
\begin{gathered}
x(\widetilde{H}(s)) \leq M \xi^{*}(t-s)^{-q \gamma-1} e^{L s} \Phi(\Omega), \\
\sup _{t \in[0, T]}\left(e^{-L t} \sup _{s \in[0, t]} x\left(\widehat{\Psi}_{2}(\Omega)(s)\right)\right) \leq M \xi^{*} \sup _{t \in[0, T]} \int_{0}^{t}(t-s)^{-q \gamma-1} e^{-L(t-s)} d s \cdot \Phi(\Omega) \\
=L_{2} \Phi(\Omega) .
\end{gathered}
$$

Now, from (3.31) and (3.34), $L>0$ can be chosen so that

$$
\Phi(\widehat{\mathcal{F}}(\Omega)) \leq\left(L_{1}+L_{2}\right) \Phi(\Omega)=\tilde{L} \Phi(\Omega),
$$

where $0<\widetilde{L}<1$.

From (3.25), we have $\Phi(\Omega)=0$. Next, we will prove that $\bmod _{c}(\Omega)=0$.

Let $\delta>0, t_{1}, t_{2} \in(0, T]$ such that $0<t_{2}-t_{1} \leq \delta$ and $v \in \Omega$, noting that (Hf)(1) and $(\mathrm{Hg})(1)$, we obtain

$$
\begin{aligned}
& \| \int_{0}^{t_{1}}\left(t_{1}-s\right)^{q-1} p_{q}\left(t_{1}-s\right)\left[f\left(s, v_{s}+\widehat{\phi}_{s}\right)+a(v+\widehat{\phi})(s)\right] d s \\
& \quad-\int_{0}^{t_{2}}\left(t_{2}-s\right)^{q-1} p_{q}\left(t_{2}-s\right)\left[f\left(s, v_{s}+\widehat{\phi}_{s}\right)+a(v+\widehat{\phi})(s)\right] d s \| \\
& \leq\left\|\int_{0}^{t_{1}}\left[\left(t_{2}-s\right)^{q-1}-\left(t_{1}-s\right)^{q-1}\right] p_{q}\left(t_{2}-s\right)\left[f\left(s, v_{s}+\widehat{\phi}_{s}\right)+a(v+\widehat{\phi})(s)\right] d s\right\| \\
& \quad+\left\|\int_{t_{1}}^{t_{2}}\left(t_{2}-s\right)^{q-1} p_{q}\left(t_{2}-s\right)\left[f\left(s, v_{s}+\widehat{\phi}_{s}\right)+a(v+\widehat{\phi})(s)\right] d s\right\| \\
& \quad+\left\|\int_{0}^{t_{1}}\left(t_{1}-s\right)^{q-1}\left[p_{q}\left(t_{2}-s\right)-p_{q}\left(t_{1}-s\right)\right]\left[f\left(s, v_{s}+\widehat{\phi}_{s}\right)+a(v+\widehat{\phi})(s)\right] d s\right\| \\
& =I_{1}+I_{2}+I_{3} .
\end{aligned}
$$

Using (2.23), (3.13), and (3.14), we have

$$
I_{1} \leq \alpha M \int_{0}^{t_{1}}\left|\left(t_{2}-s\right)^{q-1}-\left(t_{1}-s\right)^{q-1}\right|\left(t_{2}-s\right)^{-q(1+\gamma)}\left[\mu(s)+m^{*}\right] d s .
$$

Clearly, $I_{1}$ tends to zero as $t_{2} \rightarrow t_{1}$. Similarly, for $I_{2}$, we have

$$
I_{2} \leq \alpha M \int_{t_{1}}^{t_{2}}\left(t_{2}-s\right)^{-q \gamma-1}\left[\mu(s)+m^{*}\right] d s \longrightarrow 0, \quad \text { as } t_{2} \longrightarrow t_{1} .
$$


For $I_{3}$, for $\varepsilon>0$ small enough, noting that (2.23), (3.13), and (3.14), we have

$$
\begin{aligned}
I_{3} \leq & \alpha \int_{0}^{t_{1}-\varepsilon}\left(t_{1}-s\right)^{q-1}\left\|p_{q}\left(t_{2}-s\right)-p_{q}\left(t_{1}-s\right)\right\|_{L(X)}\left[\mu(s)+m^{*}\right] d s \\
& +\alpha \int_{t_{1}-\varepsilon}^{t_{1}}\left(t_{1}-s\right)^{q-1}\left\|p_{q}\left(t_{2}-s\right)-p_{q}\left(t_{1}-s\right)\right\|_{L(X)}\left[\mu(s)+m^{*}\right] d s \\
\leq & \alpha \sup _{s \in\left[0, t_{1}-\varepsilon\right]}\left\|D_{q}\left(t_{2}-s\right)-p_{q}\left(t_{1}-s\right)\right\|_{L(X)} \cdot \int_{0}^{t_{1}-\varepsilon}\left(t_{1}-s\right)^{q-1}\left[\mu(s)+m^{*}\right] d s \\
& +\alpha M \int_{t_{1}-\varepsilon}^{t_{1}}\left(\frac{\left(t_{1}-s\right)^{q-1}}{\left(t_{1}-s\right)^{q(\gamma+1)}}+\frac{\left(t_{1}-s\right)^{q-1}}{\left(t_{2}-s\right)^{q(\gamma+1)}}\right)\left[\mu(s)+m^{*}\right] d s,
\end{aligned}
$$

it follows from Theorem 2.18 that $I_{3}$ tends to zero as $t_{2} \rightarrow t_{1}$ and $\varepsilon \rightarrow 0$.

For the case when $0=t_{1}<t_{2} \leq T$, we can see

$$
\begin{gathered}
\left\|\int_{0}^{t_{2}}\left(t_{2}-s\right)^{q-1} p_{q}\left(t_{2}-s\right)\left[f\left(s, v_{s}+\widehat{\phi}_{s}\right)+a(v+\widehat{\phi})(s)\right] d s\right\| \\
\quad \leq \alpha M \int_{0}^{t_{2}}\left(t_{2}-s\right)^{-q \gamma-1}\left[\mu(s)+m^{*}\right] d s \longrightarrow 0, \quad \text { as } t_{2} \longrightarrow 0 .
\end{gathered}
$$

Thus, the set $\{(\hat{\mathscr{F}} v)(\cdot): v \in \Omega\}$ is equicontinuous, then $\bmod _{c}(\widehat{\mathscr{F}} \Omega)=0$. From (3.25), we get that $\bmod _{c}(\Omega)=0$. Hence $v(\Omega)=(0,0,0)$.

The regularity property of $v$ implies the relative compactness of $\Omega$. Now, it follows from Definition 2.7 that $\hat{\boldsymbol{F}}$ is $v$-condensing.

Consider the set

$$
B_{\rho}=\left\{v \in \mathcal{C}:\|v\|_{[0, T]} \leq \rho\right\} .
$$

Next, we show that there exists some $\rho>0$ such that $\widehat{\mathscr{F}} B_{\rho} \subset B_{\rho}$. Suppose on the contrary that for each $\rho>0$ there exist $v^{\rho}(\cdot) \in B_{\rho}$ and some $t \in[0, T]$ such that $\left\|\left(\widehat{\mathscr{F}} v^{\rho}\right)(t)\right\|_{[0, T]}>\rho$.

Noting the Hölder inequality, we have

$$
\int_{0}^{t}(t-s)^{-1-q \gamma} \mu(s) d s \leq t^{(-(1+p q \gamma)) / p} l_{p, q}\|\mu\|_{L^{p}} \leq T^{(-(1+p q r)) / p} l_{p, q}\|\mu\|_{L^{p}} .
$$


By (2.23), (Hf)(1), (Hg)(1), and (3.42), we have

$$
\begin{aligned}
& \left\|\left(\widehat{\bar{f}} v^{\rho}\right)(t)\right\| \\
& \quad=\left\|\int_{0}^{t}(t-s)^{q-1} p_{q}(t-s)\left[f\left(s, v_{s}^{\rho}+\widehat{\phi}_{s}\right)+a\left(v^{\rho}+\widehat{\phi}\right)(s)\right] d s\right\| \\
& \quad \leq M\left[\left\|v^{\rho}\right\|_{[0, T]}+\widetilde{M}\right] \int_{0}^{t}(t-s)^{-q \gamma-1}\left[\mu(s)+m^{*}\right] d s \\
& \quad \leq M(\rho+\widetilde{M}) \cdot\left[l_{p, q} T^{(-(1+p q \gamma)) / p}\|\mu\|_{L^{p}}+\frac{-m^{*} T^{-q \gamma}}{q \gamma}\right]
\end{aligned}
$$

where $\widetilde{M}=\|\phi\|_{[-r, 0]}+\sup _{t \in[0, T]}\left\|\mathcal{S}_{q}(t) \phi(0)\right\|$.

Then,

$$
\rho<\left\|\left(\widehat{\mathscr{F}} v^{\rho}\right)(t)\right\|_{[0, T]} \leq M[\rho+\widetilde{M}] \cdot\left[l_{p, q} T^{-(1+p q \gamma) / p}\|\mu\|_{L^{p}}+\frac{-m^{*} T^{-q \gamma}}{q \gamma}\right]
$$

Dividing both sides of (3.44) by $\rho$, and taking $\rho \rightarrow \infty$, we have

$$
M\left(l_{p, q} T^{-(1+p q r) / p}\|\mu\|_{L^{p}}+\frac{-m^{*} T^{-q \gamma}}{q \gamma}\right) \geq 1
$$

This contradicts (3.5). Hence for some positive number $\rho, \widehat{q} B_{\rho} \subset B_{\rho}$. According to Theorem 2.8, problem (1.1) has at least one mild solution.

Next, for $c \in(0,1]$, we consider the following one-parameter family of maps:

$$
\begin{gathered}
\mathscr{H}:[0,1] \times \mathcal{C} \longrightarrow \mathcal{C} \\
(c, v) \longrightarrow \mathscr{H}(c, v)=c \hat{\mathscr{F}}(v) .
\end{gathered}
$$

We will demonstrate that the fixed point set of the family $\mathscr{L}$ :

$$
\text { Fix } \mathscr{H}=\{v \in \mathscr{H}(c, v) \text { for some } c \in(0,1]\}
$$


is a priori bounded. In fact, let $v \in$ Fix $\mathscr{d}$, for $t \in[0, T]$, we have

$$
\begin{aligned}
\|v(t)\| & \leq \int_{0}^{t}\left\|(t-s)^{q-1} p_{q}(t-s)\left[f\left(s, v_{s}+\widehat{\phi}_{s}\right)+a(v+\widehat{\phi})(s)\right]\right\| d s \\
& \leq M \int_{0}^{t}(t-s)^{-q \gamma-1}\left[\mu(s)+m^{*}\right] \cdot\left[\sup _{\tau \in[0, s]}\|v(\tau)\|+\widetilde{M}\right] d s \\
& \leq M\left[\sup _{s \in[0, t]}\|v(s)\| \int_{0}^{t}(t-s)^{-q \gamma-1} \mu(s) d s+m^{*} \int_{0}^{t}(t-s)^{-q \gamma-1} \sup _{\tau \in[0, s]}\|v(\tau)\| d s\right]+a_{1} \\
& \leq a_{1}+a_{2} \sup _{s \in[0, t]}\|v(s)\|+a_{3} \int_{0}^{t}(t-s)^{-q \gamma-1} \sup _{\tau \in[0, s]}\|v(\tau)\| d s,
\end{aligned}
$$

where

$$
\begin{aligned}
& a_{1}=M \widetilde{M} \cdot\left(l_{p, q} T^{-(1+p q r) / p}\|\mu\|_{L^{p}}+\frac{-m^{*} T^{-q \gamma}}{q \gamma}\right), \\
& a_{2}=M l_{p, q} T^{-(1+p q \gamma) / p}\|\mu\|_{L^{p}} \\
& a_{3}=M m^{*} .
\end{aligned}
$$

We denote that $x(t):=\sup _{s \in[0, t]}\|v(s)\|$. Let $\tilde{t} \in[0, t]$ such that $x(t)=\|v(\tilde{t})\|$. Then, by (3.48), we can see

$$
x(t) \leq a_{1}+a_{2} x(t)+a_{3} \int_{0}^{t}(t-s)^{-q \gamma-1} x(s) d s .
$$

By Lemma 2.10, there exists a constant $\mathcal{\kappa}$ such that

$$
x(t) \leq \frac{a_{1}}{1-a_{2}}+\frac{\kappa a_{1} a_{3}}{\left(1-a_{2}\right)^{2}} \int_{0}^{t}(t-s)^{-q \gamma-1} d s \leq \frac{a_{1}}{1-a_{2}}-\frac{\kappa a_{1} a_{3} T^{-q \gamma}}{q \gamma\left(1-a_{2}\right)^{2}}:=\varpi .
$$

Hence, $\sup _{t \in[0, T]}\|v(t)\| \leq \varpi$.

Now, we consider a closed ball as follows:

$$
B_{R}=\left\{v \in \mathcal{C}:\|v\|_{[0, T]} \leq R\right\} \subset \mathcal{C} .
$$

We take the radius $R>0$ large enough to contain the set Fix $\mathscr{H}$ inside itself. Moreover, from the proof above-mentioned, $\hat{f}: B_{R} \rightarrow \mathcal{C}$ is $v$-condensing, and it remains to apply Theorem 2.9. 


\section{Application}

Example 4.1. Let

$$
\widehat{A}=(-i \Delta+\sigma)^{1 / 2}, \quad D(\widehat{A})=W^{1,3}\left(\mathbf{R}^{2}\right) \quad(\text { a Sobolev space })
$$

be as in [15, Example 6.3], in which, the authors demonstrate that $\widehat{A} \in \Theta_{\omega}^{\gamma}\left(L^{3}\left(\mathbf{R}^{2}\right)\right)$ for some $0<\omega<\pi / 2$ and $\gamma=-1 / 6$. We denote the semigroup associated with $\widehat{A}$ by $T(t)$ and $\|T(t)\| \leq$ $C_{0} t^{-5 / 6}\left(C_{0}\right.$ is a constant $)$.

Let $X=L^{3}\left(\mathbf{R}^{2}\right)$, we consider the following integrodifferential problem:

$$
\begin{aligned}
\partial_{t}^{q} w(t, x)= & \widehat{A} w(t, x)+\frac{1}{\sqrt[2 k]{t}} \int_{0}^{t}(t-s)^{(1 /(2 k))-1} \int_{-r}^{0} \zeta_{1}(\theta) \cdot \sin \left(\left|w_{t}(\theta, x)\right| s^{k}\right) d \theta d s \\
& +\int_{0}^{t}(t-s)^{k} \int_{-r}^{0} \zeta_{2}(\theta) w_{s}(\theta, x) d \theta d s, \\
w(\theta, x)= & w_{0}(\theta, x), \quad-r \leq \theta \leq 0,
\end{aligned}
$$

where $\partial_{t}^{q}$ is the Caputo fractional partial derivative of order $0<q<1, t \in(0,1], r>0, x \in \mathbf{R}^{2}$ and $w_{t}(\theta, x)=w(t+\theta, x) \cdot \zeta_{i}:[-r, 0] \rightarrow \mathbf{R}(i=1,2)$, and $\int_{-r}^{0}\left|\zeta_{i}(\theta)\right| d \theta<\infty(i=1,2), k>1$ is a constant.

For $x \in \mathbf{R}^{2}$ and $\varphi \in C([-r, 0], X)$, we set

$$
\begin{gathered}
u(t)(x)=w(t, x), \\
\phi(\theta)(x)=w_{0}(\theta, x), \quad \theta \in[-r, 0], \\
f(t, \varphi)(x)=\frac{1}{\sqrt[2 k]{t}} \int_{0}^{t}(t-s)^{(1 /(2 k))-1} \int_{-r}^{0} \zeta_{1}(\theta) \cdot \sin \left(|\varphi(\theta)(x)| s^{k}\right) d \theta d s, \\
g(t, s, \varphi)(x)=(t-s)^{k} \int_{-r}^{0} \zeta_{2}(\theta) \varphi(\theta)(x) d \theta .
\end{gathered}
$$

Then the above equation (4.2) can be reformulated as the abstract (1.1).

For $t \in(0,1]$, we can see that

$$
\|f(t, \varphi)\| \leq \frac{1}{\sqrt[2 k]{t}}\left(\int_{0}^{t}(t-s)^{(1 /(2 k))-1} s^{k} d s \cdot \int_{-r}^{0}\left|\zeta_{1}(\theta)\right| d \theta\right) \cdot\|\varphi\|_{[-r, 0]}:=\mu(t) \cdot\|\varphi\|_{[-r, 0]},
$$

clearly, $\mu(t)=t^{k} B(1 /(2 k), k+1) \int_{-r}^{0}\left|\zeta_{1}(\theta)\right| d \theta \in L^{p}\left([0,1], \mathbf{R}^{+}\right)(p>6 / q)$, where $B(\cdot, \cdot)$ is a beta function. 
For any $\varphi, \tilde{\varphi} \in C([-r, 0], X)$,

$$
\|f(t, \varphi)(x)-f(t, \tilde{\varphi})(x)\| \leq \frac{1}{\sqrt[2 k]{t}} \int_{0}^{t}(t-s)^{(1 /(2 k))-1} s^{k} d s \cdot \int_{-r}^{0}\left|\zeta_{1}(\theta)\right|\|\varphi(\theta)(x)-\tilde{\varphi}(\theta)(x)\| d \theta
$$

Therefore, for any bounded set $D \subset C([-r, 0], X)$, we have

$$
x(f(t, D)) \leq \mu(t) \sup _{-r \leq \theta \leq 0} x(D(\theta)), \quad \text { a.e. } t \in[0,1] .
$$

Moreover, for almost all $(t, s) \in \Lambda$ :

$$
\|g(t, s, \varphi)(x)\|=\left\|(t-s)^{k} \int_{-r}^{0} \zeta_{2}(\theta) \varphi(\theta)(x) d \theta\right\| \leq m(t, s)\|\varphi\|_{[-r, 0]},
$$

where $m(t, s):=(t-s)^{k} \int_{-r}^{0}\left|\zeta_{2}(\theta)\right| d \theta$. Then, we obtain

$$
\begin{gathered}
m^{*}=\sup _{t \in[0,1]} \int_{0}^{t} m(t, s) d s=\int_{-r}^{0}\left|\zeta_{2}(\theta)\right| d \theta \cdot \sup _{t \in[0,1]} \int_{0}^{t}(t-s)^{k} d s=\frac{1}{k+1} \int_{-r}^{0}\left|\zeta_{2}(\theta)\right| d \theta \\
\|g(t, s, \varphi)(x)-g(t, s, \tilde{\varphi})(x)\| \leq(t-s)^{k} \int_{-r}^{0}\left|\zeta_{2}(\theta)\right|\|\varphi(\theta)(x)-\tilde{\varphi}(\theta)(x)\| d \theta .
\end{gathered}
$$

Hence, for any bounded set $D \subset C([-r, 0], X)$, we have

$$
\chi(g(t, s, D)) \leq \xi(t, s) \sup _{-r \leq \theta \leq 0} \chi(D(\theta)),
$$

where $\xi(t, s):=(t-s)^{k} \int_{-r}^{0}\left|\zeta_{2}(\theta)\right| d \theta$, and $\sup _{t \in[0,1]} \int_{0}^{t} \xi(t, s) d s=1 /(k+1) \int_{-r}^{0}\left|\zeta_{2}(\theta)\right| d \theta$.

Suppose further that there exists constant $M^{*} \in(0,1)$ such that

$$
\frac{q C_{0} \Gamma(1-\gamma)}{\Gamma(1-q \gamma)}\left(l_{p, q}\|\mu\|_{L^{p}}+\frac{-m^{*}}{q \gamma}\right)<M^{*}<1
$$

then for $\phi(0) \in D\left(\widehat{A}^{\beta}\right)(\beta>5 / 6)$, problem (4.2) has at least a mild solution by Theorem 3.1.

\section{Acknowledgment}

This work is supported by Educational Commission of Yunnan Province (22012Z010) and the NSF of Yunnan Province (2009ZC054M). 


\section{References}

[1] M. M. El-Borai, "Some probability densities and fundamental solutions of fractional evolution equations," Chaos, Solitons \& Fractals, vol. 149, pp. 823-831, 2004.

[2] L. Hu, Y. Ren, and R. Sakthivel, "Existence and uniqueness of mild solutions for semilinear integrodifferential equations of fractional order with nonlocal initial conditions and delays," Semigroup Forum, vol. 79, no. 3, pp. 507-514, 2009.

[3] F. Li, "Solvability of nonautonomous fractional integrodifferential equations with infinite delay," Advances in Difference Equations, vol. 2011, Article ID 806729, 18 pages, 2011.

[4] F. Li, "An existence result for fractional differential equations of neutral type with infinite delay," Electronic Journal of Qualitative Theory of Differential Equations, no. 52, pp. 1-15, 2011.

[5] F. Li, T. J. Xiao, and H. K. Xu, "On nonlinear neutral fractional integrodifferential inclusions with infinite delay," Journal of Applied Mathematics, vol. 2012, Article ID 916543, 19 pages, 2012.

[6] F. Mainardi, P. Paradisi, and R. Gorenoo, "Probability distributions generated by fractional diffusion equations," in Econophysics: An Emerging Science, J. Kertesz and I. Kondor, Eds., Kluwer, Dordrecht, The Netherlands, 2000.

[7] K. S. Miller and B. Ross, An Introduction to the Fractional Calculus and Differential Equations, A WileyInterscience Publication, John Wiley \& Sons, New York, NY, USA, 1993.

[8] G. M. Mophou and G. M. N'Guérékata, "Existence of the mild solution for some fractional differential equations with nonlocal conditions," Semigroup Forum, vol. 79, no. 2, pp. 315-322, 2009.

[9] G. M. Mophou and G. M. N'Guérékata, "Existence of mild solutions of some semilinear neutral fractional functional evolution equations with infinite delay," Applied Mathematics and Computation, vol. 216, no. 1, pp. 61-69, 2010.

[10] G. M. Mophou and G. M. N'Guérékata, "A note on a semilinear fractional differential equation of neutral type with infinite delay," Advances in Difference Equations, vol. 2010, Article ID 674630, 8 pages, 2010.

[11] I. Podlubny, Fractional Differential Equations, vol. 198 of Mathematics in Science and Engineering, Academic Press, San Diego, Calif, USA, 1999.

[12] V. Obukhovskii and J.-C. Yao, "Some existence results for fractional functional differential equations," Fixed Point Theory, vol. 11, no. 1, pp. 85-96, 2010.

[13] G. M. N'Guérékata, "A Cauchy problem for some fractional abstract differential equation with non local conditions," Nonlinear Analysis: Theory, Methods \& Applications, vol. 70, no. 5, pp. 1873-1876, 2009.

[14] Y. Ren, Y. Qin, and R. Sakthivel, "Existence results for fractional order semilinear integro-differential evolution equations with infinite delay," Integral Equations and Operator Theory, vol. 67, no. 1, pp. 3349, 2010.

[15] R.-N. Wang, D.-H. Chen, and T.-J. Xiao, “Abstract fractional Cauchy problems with almost sectorial operators," Journal of Differential Equations, vol. 252, no. 1, pp. 202-235, 2012.

[16] J. Liang and T. J. Xiao, "Functional-differential equations with infinite delay in Banach spaces," International Journal of Mathematics and Mathematical Sciences, vol. 14, no. 3, pp. 497-508, 1991.

[17] J. Liang, T.-J. Xiao, and J. van Casteren, "A note on semilinear abstract functional differential and integrodifferential equations with infinite delay," Applied Mathematics Letters, vol. 17, no. 4, pp. 473 $477,2004$.

[18] J. Liang and T.-J. Xiao, "Solvability of the Cauchy problem for infinite delay equations," Nonlinear Analysis: Theory, Methods E Applications, vol. 58, no. 3-4, pp. 271-297, 2004.

[19] T.-J. Xiao and J. Liang, The Cauchy Problem for Higher Order Abstract Differential Equations, vol. 1701 of Lecture Notes in Mathematics, Springer, Berlin, Germany, 1998.

[20] T.-J. Xiao and J. Liang, "Blow-up and global existence of solutions to integral equations with infinite delay in Banach spaces," Nonlinear Analysis: Theory, Methods E Applications, vol. 71, no. 12, pp. e1442e1447, 2009.

[21] W. von Wahl, "Gebrochene Potenzen eines elliptischen Operators und parabolische Differentialgleichungen in Räumen hölderstetiger Funktionen," Nachrichten der Akademie der Wissenschaften in Göttingen. II. Mathematisch-Physikalische Klasse, vol. 11, pp. 231-258, 1972.

[22] R. R. Akhmerov, M. I. Kamenskiř, A. S. Potapov, A. E. Rodkina, and B. N. Sadovski1̌, Measures of Noncompactness and Condensing Operators, vol. 55 of Operator Theory: Advances and Applications, Birkhäuser, Basel, Switzerland, 1992.

[23] M. Kamenskii, V. Obukhovskii, and P. Zecca, Condensing Multivalued Maps and Semilinear Differential Inclusions in Banach Spaces, vol. 7 of De Gruyter Series in Nonlinear Analysis and Applications, Walter de Gruyter \& Co., Berlin, Germany, 2001. 
[24] D. Henry, Geometric Theory of Semilinear Parabolic Partial Differential Equations, Springer, Berlin, Germany, 1989.

[25] M. Haase, The Functional Calculus for Sectorial Operators, vol. 169 of Operator Theory: Advances and Applications, Birkhäuser, Basel, Switzerland, 2006.

[26] F. Periago and B. Straub, "A functional calculus for almost sectorial operators and applications to abstract evolution equations," Journal of Evolution Equations, vol. 2, no. 1, pp. 41-68, 2002. 


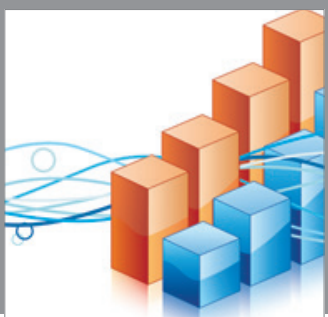

Advances in

Operations Research

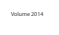

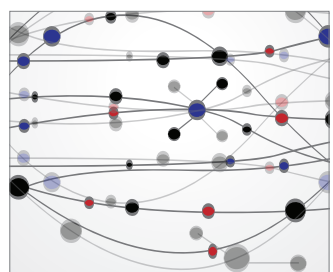

\section{The Scientific} World Journal
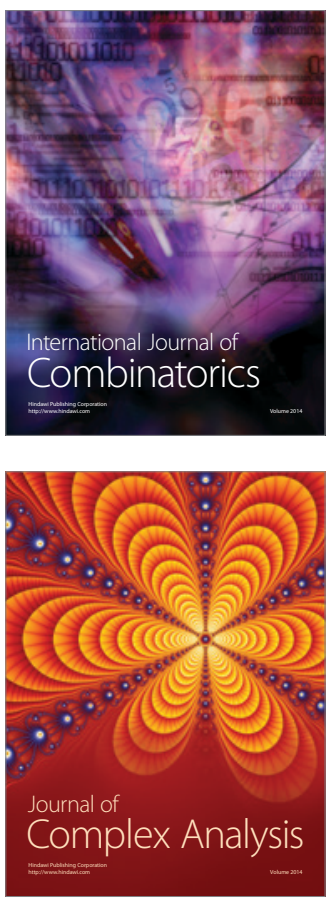

International Journal of

Mathematics and

Mathematical

Sciences
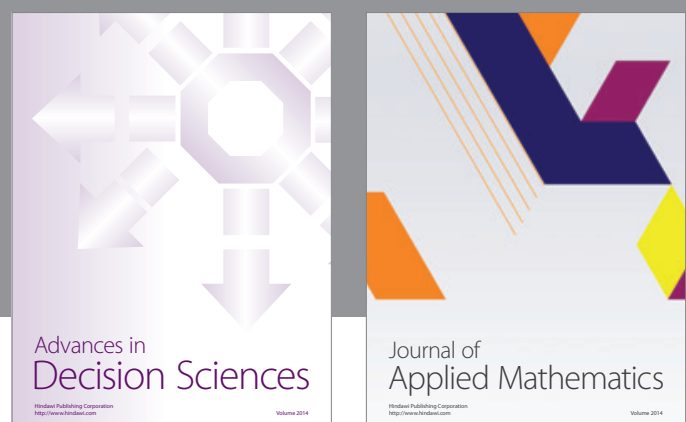

Journal of

Applied Mathematics
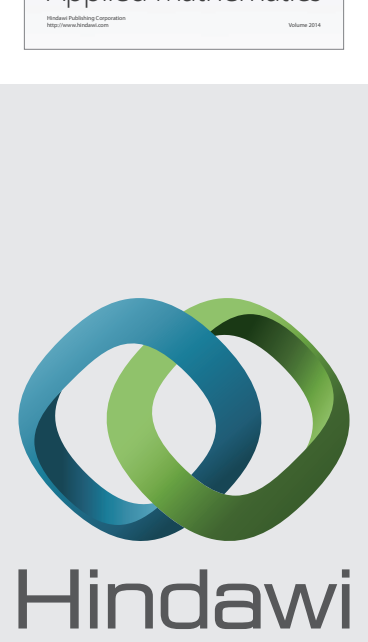

Submit your manuscripts at http://www.hindawi.com
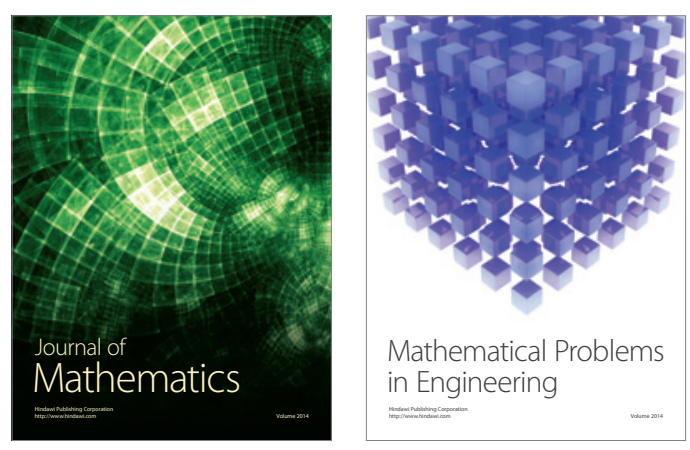

Mathematical Problems in Engineering
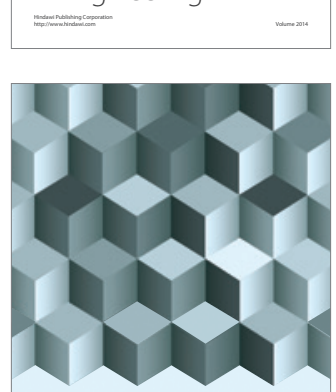

Journal of

Function Spaces
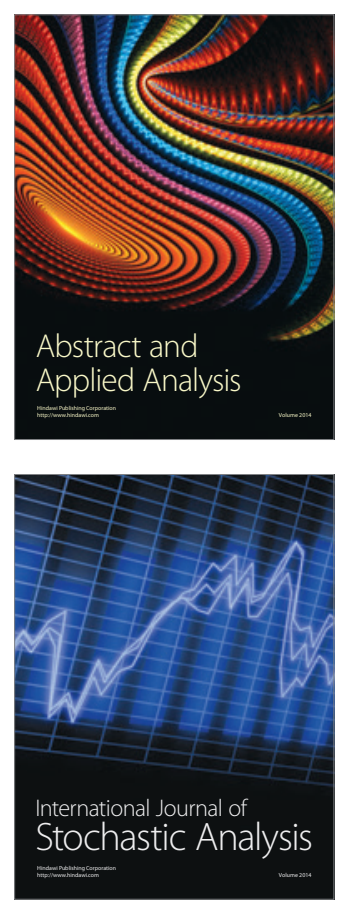

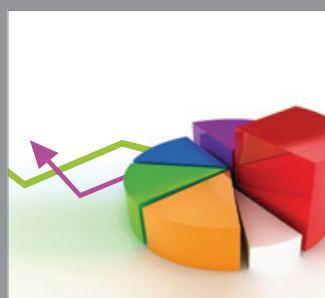

ournal of

Probability and Statistics

Promensencen
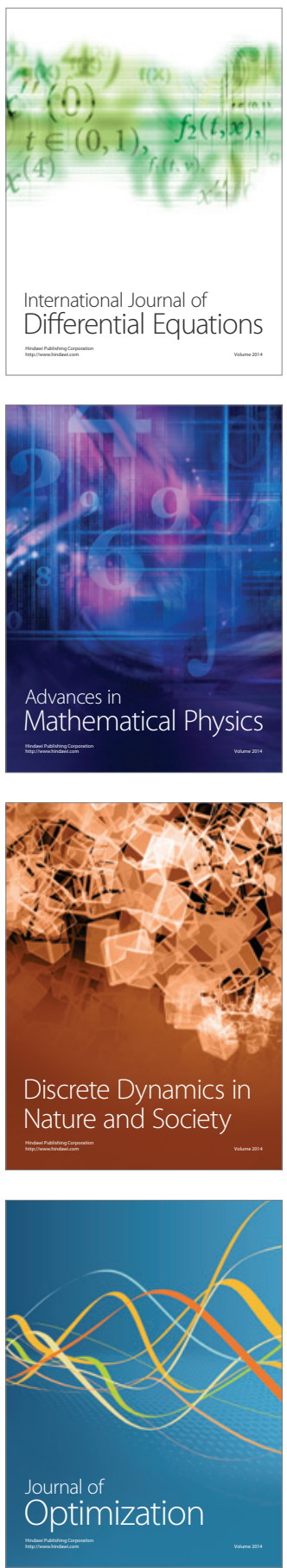